\title{
TRATAMENTO DO PARKINSONISMO COM L-DOPA
}

\author{
Nilton Luís LatuF *: \\ Marcus J. Colbachini क: \\ José A. BRaz Galvão : :
}

Várias foram as etapas no tratamento da moléstia de Parkinson, iniciado pràticamente em $1874 \mathrm{com}$ os solanáceos por Charcot ${ }^{6}$. Desde então muitas drogas foram empregadas com resultados pouco satisfatórios, tendo em vista os principais sintomas desta moléstia, ou seja, o tremor, a rigidez e a bradicinesia. Algumas esperanças surgiram com o advento da cirurgia estereotáxica, em 1947, sobretudo quanto ao tremor.

Nova era surgiu com a descoberta da dopamina no encéfalo em 1957, sendo demonstrada sua localização preferencial no corpo estriado, substância negra e globo pálido. Por outro lado, foi verificado que, no cérebro dos pacientes com moléstia de Parkinson, havia uma redução de aminas biogênicas, serotonina, noradrenalina e sobretudo, de dopamina ${ }^{11}$. De grande significado foram as observações mostrando a redução da dopamina no hemisfério contralateral, de pacientes com hemiparkinsonismo ${ }^{11} \mathrm{e}$ a diminuição de excreção urinária de dopamina nestes pacientes.

Com base nestes achados Hornyewicz ${ }^{11}$, Barbeau e col. ${ }^{2}$, Gerstenbrand e col. ${ }^{10}$, entre outros, administraram precursores da dopamina por via endovenosa; entretanto esta substância não atravessa a barreira hemoliquórica e os resultados foram discrepantes, com melhoras transitórias, particularmente da bradicinesia, ocorrendo efeitos indesejáveis, como náuseas e vômitos. Ulteriormente Cotzias ${ }^{7}$ e outros, administrando doses elevadas de DL-Dopa e, em seguida, de L-Dopa, obtiveram resultados definitivamente favoráveis, tendo verificado também serem menores os efeitos tóxicos com a L-Dopa do que com a mistura racêmica. Desde então vários autores relataram resultados favoráveis em mais ou menos $70 \%$ dos pacientes, sobretudo no que diz respeito à bradicinesia e rigidez. São descritos efeitos colaterais como náuseas, vômitos, anorexia, hipotensão ortostática, arritmias cardíacas, cefaléias, hipercinesias córeo-atetóticas, agitação, insônia e distúrbios psíquicos, assim como leucopenia e alterações da azotemia.

O propósito dêste estudo é trazer a nossa colaboração decorrente da experiência haurida no tratamento de 54 pacientes parkinsonianos com L-Dopa.

Seccão de Neurocirurgia Santa Casa de Misericórdia de Ribeirăo Prêto: * Chefe do Serviço; ** Médicos estagiários. 


\section{MATERIAL E METTODOS}

Iniciamos o tratamento do parkinsonismo com L-Dopa em março de 1970, contando a nossa experiência até a presente data com 70 casos. Entretanto serão considerados apenas 54 casos, todos com tempo de tratamento indo do minimo de 3 meses até 16 meses. O parkinsonismo era de causa idiopática em 51 casos, pósencefalítico em dois casos e pós-traumático em um caso. Quanto à idade, o paciente mais jovem tinha 39 anos e, o mais idoso, 77 anos de idade; 27 pacientes eram do sexo masculino e, 27, do sexo feminino.

o tempo de duração da moléstia variou entre 3 meses até 44 anos. O sintoma inicial mais comum foi o tremor $(87,2 \%)$, seguido pela rigidez $(9,2 \%)$ e bradicinesia $(3,6 \%)$. Apenas dois pacientes haviam sofrido intervencão estereotáxica. O grau de intensidade do tremor, da rigidez e da bradicinesia foi avaliado atribuindo notas de 0 a 5 dadas pelo mesmo observador, antes e depois da administração da droga. Isto foi feito por não contarmos com métodos de medida objetiva como os usados por Nashold ${ }^{14}$, Boshes ${ }^{4}$, Barbeau ${ }^{3}$ e Martinez ${ }^{13}$. A capacidade funcional foi verificada mediante a escala NUDS (Northwestern University Disability Scales) ${ }^{\circ}$, sendo conferidas notas de 0 a 10 , sempre pelo mesmo observador, para a marcha, ato de vestir-se, hábitos de higiene, linguagem e capacidade de nutrição. A escrita também foi avaliada antes e durante o tratamento.

Em 28 casos o tratamento inicial foi realizado com os pacientes hospitalizados durante 10 dias, sendo os pacientes restantes medicados em ambulatório. Em todos os casos foram feitas, antes e durante o estudo, exame hematológico, teste de Coombs direto, dosagens de uréia, ácido úrico, creatinina, colesterol e glicose no sangue, exame de rotina de urina, eletrencefalograma e eletrocardiograma. o seguimento foi realizado em ambulatório com intervalos de 15 ou 30 dias.

Dos 54 pacientes, 18 não tomavam qualquer medicação prévia; dos demais a medicação que vinha sendo utilizada foi retirada paulatinamente, à medida que eram aumentadas as doses de L-Dopa. O plano inicial foi de realizarmos o estudo "duplo cego"; entretanto a melhora dos pacientes foi tão evidente que consideramos êste cuidado como desnecessário.

Iniciamos o tratamento com a administraçao diária de $750 \mathrm{mg}$ de L-Dopa *, dividida em 3 vêzes às 8 , às 12 e às 16 horas, aumentando $250 \mathrm{mg}$ no 3.0 dia às 20 horas e passando para total de $1.250 \mathrm{mg}$ no $4.0 \mathrm{dia}, 1.500 \mathrm{mg}$ no 5.0 dia, $1.750 \mathrm{mg}$ no 6.0 dia, $2.000 \mathrm{mg}$ no $7.0 \mathrm{dia}, 2.500 \mathrm{mg}$ no $8.0 \mathrm{dia}, 3.000 \mathrm{mg}$ no $9.0 \mathrm{dia}, 3.500 \mathrm{mg}$ no 10.0 dia e, assim por diante, até que aparecessem sinais de intolerância pela droga, sendo então a última considerada como dose limite. A dose de manutencão mínima foi de $1,5 \mathrm{~g}$ e, a máxima, de $7 \mathrm{~g}$ ao dia. Em todos os casos foi aconselhada a ingestão do medicamento após alimentação para evitar a ocorrência de náuseas ou vômitos que, em vários casos, foram controlados com antieméticos.

\section{RESULTA DOS}

Os resultados obtidos nos 54 pacientes sugerem que o medicamento atua nos principais sintomas de parkinsonismo (quadro 1). A melhora obtida em relação ao tremor foi de $61 \%$, devendo-se levar em consideração a dificuldade do observador avaliar apenas um grau de melhora. Observamos também que nos 16 casos cujo único sintoma era tremor unilateral ou não, independendo do grau de intensidade, em apenas 4 houve melhora de um grau. Por outro lado dois dos 52 casos com tremor pioraram definitivamente, o que aconteceu transitòriamente, em muitos no inicio do tratamento. Com respeito à rigidez a melhora foi de $91,9 \%$ e, quanto à bradicinesia, de $85,5 \%$, conșiderando-se a mesma observação feita acima.

\footnotetext{
* Agradecemos aos Laboratórios Lepetit o suprimento de L-Dopa (Dopalina).
} 


\begin{tabular}{lccc}
\hline \multicolumn{1}{c}{ Resultados } & Tremor & Rigidez & Bradicinesia \\
& N.0 de casos & N.o de casos & N.o de casos \\
\hline Melhora + & $27(51,9 \%)$ & $15(39,4 \%)$ & $9(25,7 \%)$ \\
Melhora ++ & $5(9,6 \%)$ & $13(34,2 \%)$ & $12(34,2 \%)$ \\
Melhora +++ & 0 & $5(13,1 \%)$ & $8(22,8 \%)$ \\
Melhora ++++ & 0 & $2(5,2 \%)$ & 0 \\
Melhora +++++ & 0 & 0 & $1(2,8 \%)$ \\
Sem melhora & $18(34,6 \%)$ & $3(7,8 \%)$ & $5(4,2 \%)$ \\
Piora & $2(3,8 \%)$ & 0 & 0 \\
Totai & 52 & 38 & 35 \\
\hline
\end{tabular}

Quadro 1 - Resultados obtidos quanto ao tremor, rigidez e bradicinesia.

Em relação à análise da capacídade funcional avaliada mediante a escala NUDS pudemos verificar que a maior variação para melhor ocorreu quanto ao ato de vestir-se, seguido da marcha, hábitos de higiene, linguagem e nutrição-alimentação (Quadro 2). Estes resultados comprovam os que foram verificados quanto ao tremor e à rigidez pois a alimentação e nutrição estão condicionadas a êstes sintomas.

\begin{tabular}{lccccc}
\hline & Vestir-se & Marcha & $\begin{array}{c}\text { Higiene } \\
\text { pessoal }\end{array}$ & Linguagem & $\begin{array}{c}\text { Nutricão } \\
\text { Alimentacão }\end{array}$ \\
Antes & 7.50 & 7.48 & 7.37 & 8.26 & 7.56 \\
Após & 8.65 & 8.61 & 8.37 & 9.09 & 8.13 \\
Variação & 1.17 & 1.13 & 1.00 & 0.83 & 0.57 \\
\hline
\end{tabular}

Quadro 2 - Diferença das médias las capacidades funcionais, antes e após o tratamento.

Apesar de todos os pacientes terem sido submetidos ao teste da escrita, assinando seu nome e fazendo círculos, foi tomada para demonstração apenas uma das pacientes (E.L.Z. - Pront. 1043) que escreveu um diário desde o inicio do tratamento, observando-se evolução para a melhora com aumento da letra e điminuição do tremor (Fig. 1).

Verificamos também que o máximo de melhora é obtldo entre o $3^{\circ}$ e 0 6.o mês de tratamento, devendo-se assinalar que, em 6 pacientes, doses inferiores mantiveram os bons resultados após êste tempo; em 36 pacientes o uso do medicamento por tempo prolongado tornou-se ineficaz durante certo periodo e, em outros (8 pa(:ientes), definitivamente.

Ejeitos colaterais - Sintomas indesejáveis, mas sem gravidade e reverssiveis, ocorreram muitas vēzes nos nossos pacientes medicados com L-Dopa. Foram observados sintomas gastro-intestinais (vômitos em 61\%, náuseas em 41\%, anorexia em 


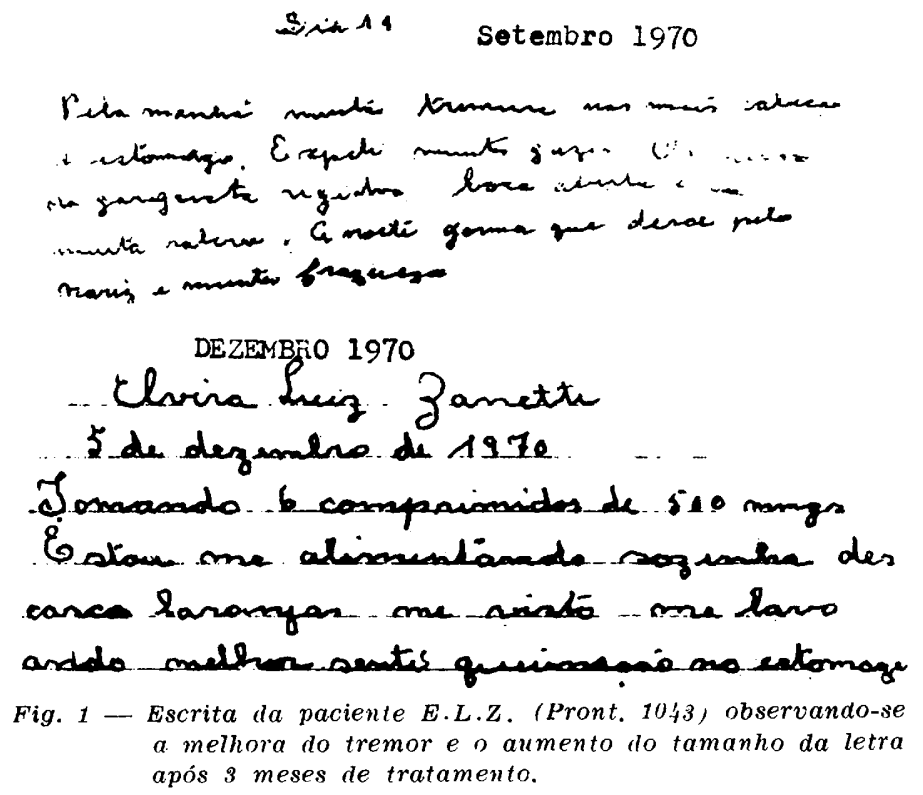

$25 \%$ e constipação intestinal em 1,80\% dos (asos), discinesias (córeo-atetoses em $14 \%$ dos casos), distúrbios psiquicos (euforia em $15 \%$, desânimo em 18\%, agitaçãoinsônia em $11 \%$ dos casos), distúrbios neuro-vegetativos (rubor em $3 \%$ ) e distúrbios cardiovaseulares em $1,80 \%$ dos casos. Com respeito aos exames laboratoriais, 6 pacientes $(11 \%)$ apresentaram leucopenia acentuada porém transitória e corrigida; linfopenia ocorreu em 6 casos $(11 \%)$, netropenia em 5 casos $(9,2 \%)$, eosinofilia em $3 \operatorname{casos}(5,5 \%)$. Não registramos alteraçōes da azotemia ou dos outros elementos pesquisados mediante exames laboratoriais e complementares.

\section{COM E N T ÁRIOS}

Estudos de vários autores (Cotzias ', Barbeau e col. ${ }^{2}$, Yahr e col. "i, Stellar e col. ${ }^{15}$, Fasano e col. ${ }^{8}$, Krayembuhl e Siegfried ${ }^{12}$ ) mostraram que de alguma forma o tratamento com L-Dopa trouxe benefícios para a maioria dos pacientes parkinsonianos. Concordamos com êstes autores, pois $61 \%$ dos nossos pacientes mostraram-se beneficiados, sobretudo quanto à rigidez e à bradicinesia.

Registramos também benefícios no que diz respeito à postura, à mímica facial, à depressão psíquica e, em menor escala à sialorréia, no que nossas observações coincidem com as de Calne e col. ${ }^{5}$ A idade dos pacientes ou o tempo de duração da moléstia não tiveram influência marcante sôbre os resultados, o que está de acôrdo com o que já foi observado ${ }^{16}$.

Dentre os efeitos colaterais predominaram os gastrointestinais como náuseas, vômitos e anorexia. Êstes sintomas apareceram sobretudo quando o medicamento era ingerido em jejum ou em doses maiores, sendo contro- 
lados mediante a ingestão após as refeiçōes ou com o uso de antieméticos de ação central. As discinesias, principalmente do tipo córeo-atetótico foram freqüentes e seu aparecimento serviu como indicador para limitação da dosagem medicamentosa e, muitas vêzes, para a fixação da posologia idea] para cada caso. Distúrbios psíquicos do tipo euforia cu agitação-insônia apareceram em pacientes mais idosos, estando provàvelmante relacionados a alterações pré-existentes; tais distúrbios foram controlados crm diazepínicos e hipnóticos mas, algumas vêzes, obrigaram à suspensão do tratamento. Observamos também um caso de intenso desânimo, também já referido por alguns autores.

Resta-nos comentar o caso de um paciente que, após 8 meses de tratamento, apresentou intensa dispnéia e taquicardia, tendo o ECG mostrado bloqueio no feixe de $\mathrm{His}$; o paciente foi medicado com quinidina após a suspensão da L-Dopa e, uma vez compensado, o tratamento pela dopamina foi reiniciado sob contrôle de cardiotônicos. Apenas dois pacientes apresantaram distúrbios neurovegetativos traduzidos for rubor.

Outros efeitos colaterais, como hipotensão ortostática, pseudo-exoftalmo, fraturas, alterações da esfera sexual, crises convulsivas, relatados por outros autores, não foram observados em nossa série. Uma das pacientes era diabética não havendo alteração da glicemia com o uso do medicamento.

Concluindo, somos levados a considerar que o L-Dopa tem real valor no tratamento do parkinsonismo, como foi demonstrado em $61 \%$ dos pacientes. Os resultados a longo prazo devem aguardar um tempo maior de observação.

\section{R E S U M O}

São apresentados os resultados obtidos no tratamento de 54 pacientes parkinsonianos com L-Dopa. A intrnsidade dos sintomas foi avaliada mediante a escala NUDS (Northwestern University Disability Scales) antes e após o tratamento. Os autores ressaltam a obtenção de melhores resultados no que respeita à rigidez e à discinesia, em comparação com o tremor. São apontados os efeitos colaterais, quase sempre de pequena intensidade e corrigiveis com a diminuição ou suspensão da administração medicamentosa.

\section{S U M M A R Y}

\section{Treatment of parkinsonism with laevodopu}

The experience gained from the treatment of 54 parkinsonian patients with laevodopa is reported. The evaluation of the tremor, rigidity and bradikinesia was done atributing grades from 0 to 5 as well as the funcional capacity by the utilization of the NUDS scale (Northwestern University Disability Scales) before and during the treatment. The administration of the drug was done progressively, the dosage being increased or reduced 
according by to the occurrence of colateral effects. The better results obtained in relation to rigidity and dyskinesia are emphasized. Collateral effects are reported.

\author{
REFERENCIAS
}

1. BARBEAU, A.; MURPHY, G. F. \& SOURKES, 'T. C. - Excretion of dopamine in diseases of basal ganglia. Science 133:1706, 1961.

2. BARBEAU, A.; SOURKES, T. L. \& MURPHY, G. F. - Les catécholamines dans la maladie de Parkinson. In Monoamines et Système Nerveux Central. J. de Ajuriaguerra ed., Genève, 1962.

3. BARBEAU, A. - The problem of measurement of akinesia. J. Neurosurg. Supl. ao vol. $24: 331,1966$.

4. BOSHES, B. - Measurement of tremor. J. Neurosurg. Supl. ao vol. 24:324, 1966.

5. CALNE, D. B.; SPIERS, A. S.; STERN, G. M. \& LAURENCE, D. R. - L-Dopa in idiopathic parkinsonism. Lancet, II:973, 1969.

6. CHARCOT, V. A. - Lecons sur les Maladies du Système Nerveux faites à la Salpêtrière. Recueillies par A. Bourneville. Delahaye et Lecrosnler, Paris, 1892.

7. Cotzias, G. C. - L-Dopa in Parkinson's disease. Hosp. Pract. 4:35, 1969.

8. FASANO, V. A.; URCIUOLI, R.; BROGGI, G.; CANNElla, M. \& LOMBARD, G. F. - Observations cliniques sur l'action de la levo-dopa por voie intraveineuse et par voie orale dans le traitement de la maladie de Parkinson. Neuro-Chirurgie 16:171, 1970.

9. GERALD, C. J.; LA TORRE, R. \& MIER, M. - A method for evaluating disability in patients with Parkinson's disease. J. nerv. ment. Dis. 133:143, 1961.

10. GERSTENBRAND, P. \& PATEISKY, K. - Uber die Wirkung von L-Dopa auf die motorischen Störungen beim Parkinson-Syndrom. Wiener Wschr. f. Nervenheilk. 20:90, 1962.

11. HORNYEWICZ, O. \& BIRKMAYER, W. - Der L-3, 4 - dioxyphennyl alanin (L-Dopa): Effekt bei der Parkinson-Akineses. Wien Klin. Wschr. 73:787, 1961.

12. KRAYENBUHL, H. \& SIEGFRIED, J. - Traitement de la maladie de Parkinson: L-Dopa ou stéréotaxie? Neuro-Chirurgie 16:71, 1970.

13. MARTINEZ, N. - Measurement of rigidity with a strain gauge myokinetograph. J. Neurosurg. Supl. ao vol. 24:315, 1966.

14. NASHOLD Jr., B. S. - Measurement of tremor. J. Neurosurg. Supl. ao vol. 24:320, 1966.

15. STEllaR, S.; SNANDEll, S.; WALTZ. \& COOPER, I. S. - L-Dopa in the treatment of parkinsonism. J. Neurosurg. 32:275, 1970.

16. YAHR, M. D.; DU VOISIN, R. C.; SCHEAR, M. J.; BARRET, R. E. \& HOEHN, M. M. - Treatment of parkinsonism with laevodopa. Arch. Neurol. (Chicago) $21: 343,1969$.

Serviço de Neuracirurgia - Santa Casa de Misericórdia - Caixa Postal 644 14100 Ribeirāo Prêto SP - Brasil. 